\title{
CUR Focus \\ Creative Funding Strategies for Undergraduate Research at a Primarily Undergraduate Liberal Arts Institution
}

A s peer-reviewed research has shown (Elrod et al. 2010), involvement in a faculty-mentored culture of inquiry concerned with authentic, real-world questions, issues, or ideas can be a transformative experience for students. Given this fact, since 2009, students at Loyola Marymount University have had access to a range of programs to facilitate their engagement in scholarly research and creative activities. These include the Undergraduate Research Opportunities Program, the Summer Undergraduate Research Program, and the Undergraduate Research Symposium. Especially since the establishment of the Office of Undergraduate Research in 2012, participation in these programs has grown substantially, with approximately 10 percent of all undergraduates participating in these programs during academic 2013-2014.

As on any campus, scholarly research and creative work at LMU encompass an array of academic activities, the mix of which varies by disciplinary area. In the biological sciences, where understanding the natural world is the focus, undergraduates work in the laboratory and/or in the field, and generally conduct experiments or other data-gathering activities, followed by statistical analysis of results and the preparation of research results and conclusions for public presentation. In contrast, in engineering, where the primary goal is creation of "cost-effective solutions to practical problems by applying scientific knowledge to building things" (Shaw 1990), student work will often involve the development and prototyping of a solution, with the laboratory being used as a locale to gather experimental data needed for the validation and improvement of the solution, prior to communicating project outcomes to relevant stakeholders. Finally, in the social sciences, where understanding the human experience is the focus, students are likely to engage in a wide range of qualitative and quantitative methods of data gathering, from conducting surveys to performing database searches, prior to the analysis of research findings and the formulation of potential societal implications/recommendations for dissemination.

To support the extensive work underway across disciplines, LMU faculty members have been exploring a number of nontraditional mechanisms for gaining external support. Here we will explore how individuals cultivated partnerships with industry to support labs, leveraged interest in special events to raise funds, and engaged in contract work to provide students with practical experience.

\section{Cultivating Industry Partners}

In 2011, LMU renovated a fluid mechanics laboratory with support from federal funding and donors. The new James E. Foxworthy Fluid Dynamics Laboratory consists of 2,000 square feet devoted to experiments in fluid mechanics and hydraulics, and includes additional laboratories in materials science, thermodynamics, rapid prototyping, engineering design, hydrology, and a computer station for numerical modeling in computational fluid dynamics. The new laboratory has offered faculty members, industry representatives, donors, and students a chance to interact with each other in the new space while discussing the laboratory courses and applied research that both faculty members and undergraduate students can conduct. For example, a local firm that manufactures sensors that measure water levels in tanks became interested in using the laboratory's open channel facilities to test new sensors it was developing to measure flow in open channel weirs and flumes.

Working with a faculty member in mechanical engineering, a partnership developed that involved testing the prototype sensors under different hydraulic conditions by following carefully designed testing protocols that satisfied the company's specifications. Five undergraduates were involved in the effort, and a report summarizing the results was submitted to the company. Funding was made possible through a $\$ 10,000$ in-kind gift from the company, which was later increased by approximately $\$ 3,000$, plus donations of equipment (estimated at $\$ 5,000)$. A new undergraduate team is currently preparing for a new phase of testing, which may result in additional long-term opportunities with the company.

This project not only helped undergraduate engineering students learn the techniques and methods used in testing protocols, it also exposed them to the role of engineering within a competitive business environment, which will be an invaluable experience for our job-seeking graduates. The real-world learning opportunity for our students significantly enhanced their skills, understanding, and confidence, leading to the production of considerable undergraduate research. Within the past two years, the same weir and flume facilities have been used to generate one conference paper, two conference presentations, and four conference posters with undergraduates, and one conference paper and poster with graduate students. A paper for a top research journal also is currently being completed. 


\section{Leveraging Special Events}

The Thomas and Dorothy Leavey Center for the Study of Los Angeles, led by a faculty member in political science, has been extremely successful in fostering donations, contracts, and other forms of support through hosting an annual event that attracts a great deal of attention in the region. The center has long relied on funding from multiple sources, including university support, private donors, contracts, and sponsorships for its signature public events.

The center has successfully employed traditional funding strategies such as applying for grant funds by responding to funders' requests for proposals (RFPs). In addition, many other opportunities for funding have been explored using non-traditional methods. For example, the center at one time engaged in contract work, though it ultimately decided that this model did not work well for the kind of research staff members wished to conduct. Staff members felt that maintaining academic integrity was essential to fulfill the center's public-spirited mission, but found that difficulties arose in doing so. First, sponsors asked the center to modify the phrasing of survey questions in ways that would have led to longitudinal inconsistency and/or misleading results. Second, the center was asked to withhold publication of unflattering results. Although troubling, these experiences were extremely important for staff members and students because they provided real-world insights into some of the methodological and ethical challenges of social science research.

As a result, the center sought alternative funding methods to maintain control over the entire scope of its work. In the last decade the center successfully applied for several grants, bringing in over $\$ 350,000$. In addition, over the last five years the center has solicited funds from a variety of companies and individuals, securing nearly $\$ 500,000$ more to support its programs.

One of the signature events hosted by the center is Forecast LA, an annual conference that explores the civic and economic concerns, cultural identities, and levels of satisfaction of residents and leaders in the Los Angeles region. As part of the center's approach to forecasting, it conducts two outlook surveys: a telephone survey of adult residents in Los Angeles County and face-to-face interviews with a set of LA County leaders. Forecast LA is sponsored by dozens of corporations, municipal agencies, and other organizations, and it places LMU at the center of a vibrant community committed to a better future for Los Angeles.

At Forecast LA, joint presentations by both seasoned researchers at the center and undergraduates provide a compelling way for the funders to see what their money is supporting. This connection is also beneficial for the students because not only do they get valuable research experience and public speaking practice, but they also build up a network of connections that have proven valuable when they look for internships and jobs. After several cycles in which the center reached out to funders and then conducted and disseminated accurate and well-respected research, many companies have begun to put line items in their budgets to provide annual funding for Forecast LA, allowing center staff members to focus their efforts on securing new funding while continuing to maintain relationships with past funders.

\section{Contracts and Service-Learning Opportunities}

In addition to partnerships and event-based sponsorships, a third non-traditional approach employed at LMU involves securing contracts grounded in work by undergraduates. The renovation of the Foxworthy Labs described above provided an opportunity to partner with local industry, but it also heightened the capacity to conduct contracted research work for local municipalities as well. Two faculty members in civil and mechanical engineering combined their expertise in computational fluid dynamics (CFD) and in wastewater treatment to approach a major local agency seeking to perform CFD research on disinfection reactors at two major treatment plants that the agency operates. Funding for the project was possible through a two-phase contract totaling $\$ 42,000$. Though the agency could have hired a consulting firm at a much greater cost, it chose to build a partnership with LMU and expand its long-term relationship with one of LMU's faculty members. The contract specified that the project was to be treated as a research project in which the agency obtains the results, with LMU retaining the rights to conduct research and disseminate the results while working closely with the agency to accommodate its needs.

Contracts for student work have been particularly helpful in supporting senior capstone projects in mechanical engineering. Since the 2011-2012 academic year, industry-sponsored projects based on the Harvey Mudd Clinic Program, which has student teams complete industry-sponsored engineering projects (Bright 1994), have been offered on a scale that matches the resources and learning outcomes of LMU's mechanical engineering program. The mechanical engineering department worked with LMU's Office for Research and Sponsored Projects to create a sponsorship package; industry sponsors provide a $\$ 2,500$ donation to cover the costs of prototype fabrication. Industry-sponsored projects are primarily obtained through faculty members' relationships with alumni and with individual companies.

During academic 2012-2013, service-learning projects were also offered. The benefit of service-learning in engineering has been well documented (see Barrington and Duffy 
Finally, we have learned that nothing replaces the personal knowledge and networks of faculty members. Their engagement with alumni, with business firms and agencies, with trends in their fields, and with the kinds of learning projects that would benefit their students are the factors that most guarantee successful support for students.

\section{References}

Barrington, Linda, and John Duffy. 2010. "Maximizing Benefits of ServiceLearning in Engineering." American Society for Engineering Education (ASEE) Annual Conference and Exposition. Louisville, Kentucky.

Bright, Anthony. 1994. "Teaching and Learning in the Engineering Clinic Program at Harvey Mudd College." Journal of Engineering Education 83(2): 113116.

Elrod, Susan, Diane Husic, and Jillian Kinzie. 2010. "Research and Discovery Across the Curriculum." Peer Review 12(2): 4-8.

Shaw, Mary. 1990. "Prospects for an Engineering Discipline of Software." IEEE Software 7(6): 15-24.

Siniawski, Matthew T., Jose A. Saez, Jeremy S. Pal, and Sandra G. Luca. 2014. "Creating Learning through Service Opportunities for Engineering Students: Lessons Learned from a Primarily Undergraduate Liberal Arts University." International Journal for Service Learning in Engineering Special Issue on University Engineering Programs That Impact Communities: Critical Analyses and Reflection: 240-255.

\section{Martina Giselle Ramirez}

Loyola Marymount University, martina.ramirez@lmu.edu

Martina Giselle Ramirez is special assistant to the provost for technology-enhanced learning and professor of biology at Loyola Marymount University (LMU), Los Angeles, CA. She received her BS in biology from LMU and her PhD in biology from the University of California, Santa Cruz. Prior to LMU, Ramirez taught at Pomona College, Bucknell University, Denison University, and East Stroudsburg University. Ramirez received the Rudinica Award for Student-Faculty Research from LMU's Seaver College of Science and Engineering (2012), as well as a Biology Mentor Award from the Council on Undergraduate Research (2013).

Joseph McNicholas is director of the Office for Research and Sponsored Projects at Loyola Marymount University. He holds a doctorate and an MBA and presents at conferences and publishes regularly on research administration at predominantly undergraduate institutions. His most recent, co-authored work providing guidance for administrators is Establishing and Managing an Office for Sponsored Programs at Non-Research Intensive Colleges and Universities (NCURA 2014).
Brianne Gilbert is the associate director of the Center for the Study of Los Angeles at Loyola Marymount University. Since joining the center, Gilbert has worked on dozens of studies involving voter polls, public-opinion research, and surveys of leaders/elites. Although her specialty is quantitative methodology and analysis, she oversees all phases of the research process and has been a principal investigator on a number of projects. She has presented at regional and national conferences in sociology, anthropology, GIS (geographic information systems), education, and public-opinion research and has served as a consultant in those areas, as well as in international affairs, geology, and medicine. In addition to her work at the center, Gilbert currently teaches the Introduction to GIS course at LMU. She received her BA from Wittenberg University and her MA from Florida International University, both in sociology with emphases on methodology and statistics.

Jose A. Saez is an associate professor in the Department of Civil Engineering and Environmental Science at Loyola Marymount University. He teaches engineering courses in fluid mechanics, open channel hydraulics, water resources, water and wastewater treatment, and introduction to engineering. Saez conducts applied research in open channel hydraulics, hydrology, contaminant transport, and water and wastewater treatment. He also emphasizes the importance of engineering education and the application of engineering concepts in community service projects. Saez has $B S$ and $M S$ degrees in civil engineering from $L M U$, and a PhD from UCLA in civil engineering with emphasis on environmental engineering. Prior to joining LMU, Saez worked for sixteen years as a civil engineer with the Los Angeles County Sanitation Districts. Saez received the 2013 Rudinica Award for Teaching and Mentoring. He is also a registered civil engineer in California and consults on a part-time basis.

Matthew T. Siniawski played an instrumental role in the revitalization of the senior capstone design program for mechanicalengineering students at Loyola Marymount University, and has advised more than 40 different student capstone project teams since 2004. He has served as a principal investigator on four grants and contracts and published a total of 19 technical journal articles and proceedings and 11 industry reports and magazine articles. He has won more than $\$ 360,000$ in grants, contracts, and capstone project sponsorships. He is an active proponent of reform of engineering education and data-driven pedagogical innovation, including service learning and project-based learning and how they impact the technical and professional development of engineering undergraduates. Siniawski received his BSME from Illinois Institute of Technology and his PhD from Northwestern University in mechanical engineering. 